\title{
Comparative Study of Extracellular and Intracellular Magnetic Hyperthermia Treatments Using Magnetic Particle Imaging
}

\author{
Sayumi Kobayashi, Akiko Ohki, Minori Tanoue, Yoshimi Inaoka, Kenya Murase* \\ Department of Medical Physics and Engineering, Division of Medical Technology and Science, Faculty of Health Science, \\ Graduate School of Medicine, Osaka University, Osaka, Japan \\ Email: *murase@sahs.med.osaka-u.ac.jp
}

How to cite this paper: Kobayashi, S., Ohki, A., Tanoue, M., Inaoka, Y. and Murase, K. (2017) Comparative Study of Extracellular and Intracellular Magnetic Hyperthermia Treatments Using Magnetic Particle Imaging. Open Journal of Applied Sciences, 7, 647-660.

https://doi.org/10.4236/ojapps.2017.712047

Received: November 10, 2017

Accepted: December 4, 2017

Published: December 8, 2017

Copyright $\odot 2017$ by authors and Scientific Research Publishing Inc. This work is licensed under the Creative Commons Attribution International License (CC BY 4.0).

http://creativecommons.org/licenses/by/4.0/

(c) (i) Open Access

\begin{abstract}
The purpose of this study was to evaluate the effectiveness of intracellular magnetic hyperthermia treatment (MHT) in comparison with that of extracellular MHT using magnetic particle imaging (MPI). Colon-26 cells were implanted subcutaneously into the backs of 8 -week-old male BALB/c mice. When the tumor volume reached approximately $100 \mathrm{~mm}^{3}$, the mice were divided into control $(n=10)$, extracellular MHT $(n=8)$, and intracellular MHT groups $(n=7)$. In the control group, MHT was not performed. In the extracellular MHT and intracellular MHT groups, the tumors were injected directly with magnetic nanoparticles (MNPs) (400 mM Resovist $\left.{ }^{\oplus}\right)$ and were heated for 20 min using an alternating magnetic field. During MHT, the temperatures of the tumor and rectum were measured using optical fiber thermometers. In the extracellular MHT group, MHT was performed 15 min after the injection of MNPs, whereas MHT was performed one day after the injection of MNPs in the intracellular MHT group. In both groups, MPI images were obtained using our MPI scanner immediately before, immediately after, and 7 and 14 days after MHT. After the MPI studies, we drew a region of interest (ROI) on the tumor in the MPI image and calculated the average, maximum, and total MPI values and the number of pixels within the ROI. Transmission electron microscopic (TEM) images were also obtained from resected tumors. In all groups, tumor volume was measured every day and the relative tumor volume growth (RTVG) was calculated. The TEM images showed that almost all the MNPs were aggregated in the extracellular space in the extracellular MHT group, whereas they were contained within the intracellular space in the intracellular MHT group. Although the temperature of the tumor in the intracellular MHT group was significantly lower than that in the extracellular MHT group, the RTVG value in the intracellular MHT group was significant-
\end{abstract}


ly lower than that in the control group 2 days or more after MHT and that in the extracellular MHT group 3, 4, and 5 days after MHT. The average MPI value normalized by that immediately before MHT in the intracellular MHT group was significantly higher than that in the extracellular MHT group immediately and 7 days after MHT. The maximum and total MPI values normalized by those immediately before MHT in the intracellular MHT group were significantly higher than those in the extracellular MHT group 7 days after MHT, suggesting that the temporal change of MNPs within the tumor in the intracellular MHT group was smaller than that in the extracellular MHT group. Our results suggest that intracellular MHT is more cytotoxic than extracellular MHT in spite of a lower temperature rise of tumors, and that MPI is useful for evaluating the difference in the temporal change of MNPs in the tumor between extracellular MHT and intracellular MHT.

\section{Keywords}

Magnetic Particle Imaging (MPI), Magnetic Hyperthermia Treatment (MHT), Magnetic Nanoparticles (MNPs), Extracellular MHT, Intracellular MHT

\section{Introduction}

Magnetic hyperthermia treatment (MHT) is one of several hyperthermia treatments and utilizes the temperature rise of magnetic nanoparticles (MNPs) under an alternating magnetic field (AMF) [1]. MHT can selectively heat tumor cells without damaging normal tissues [2], unlike conventional hyperthermia treatments such as radiofrequency (RF)-capacitive heating [3]. In order to enhance the therapeutic effect of MHT, it is necessary to deliver and accumulate as many MNPs as possible into the tumor tissues [4] [5]. Furthermore, the retention of MNPs in tumors is also an important factor in enhancing the therapeutic effect of MHT [4] [5]. When considering repeated applications of MHT, it is desirable for MNPs to be retained in the tumor for as long as possible [4] [5].

Recently, it has been reported that cell death by MNPs under AMF can occur without a noticeable global increase in temperature [6] [7] [8] [9]. These results raised some debate in the literature, because it has been usually thought that it is necessary to achieve a homogeneous increase in temperature within the tumor for effective hyperthermia [10]. As a possible explanation for the above results, it has been hypothesized that even an increase in temperature localized in the vicinity of the MNPs could be enough to induce cell death without a significant global increase in temperature [6] [7] [8] [9].

Magnetic particle imaging (MPI) is an imaging method that uses the nonlinear response of MNPs to an AMF [11]. MPI is capable of imaging the spatial distribution of MNPs in positive contrast without any background and with high sensitivity and spatial resolution [11]. We recently have developed an MPI scanner based on the field-free-line encoding scheme [12] [13] and succeeded in 
imaging the intratumoral distribution of MNPs and quantifying its temporal change in vivo [14] [15]. We also showed that MPI is useful for predicting the therapeutic effect of MHT [14] [15].

This study was undertaken to compare the therapeutic effect of extracellular MHT and that of intracellular MHT in vivo using tumor-bearing mice and to discuss the difference between them based on the temporal change of MNPs in the tumor measured using MPI.

\section{Materials and Methods}

\subsection{Animal Study Protocol}

All animal studies were approved by the animal ethics committee at Osaka University School of Medicine. Seven-week-old male BALB/c mice weighing $24.3 \pm$ $1.4 \mathrm{~g}$ (mean \pm standard deviation (SD)) were purchased from Charles River Laboratories Japan, Inc. (Yokohama, Japan). After one-week habituation to the rearing environment, $1 \times 10^{6}$ cells of colon-26 (Riken Bio Resource Center, Ibaragi, Japan) were implanted into the backs of the mice [14] [15].

When the tumor volume reached approximately $100 \mathrm{~mm}^{3}$, the mice were divided into control $(n=10)$, extracellular MHT $(n=8)$, and intracellular MHT groups $(\mathrm{n}=7)$. In this study, we call the MHT performed immediately (15 min) and one day after the injection of MNPs "extracellular MHT" and "intracellular MHT", respectively.

In the control group, MHT was not performed. The tumors in the extracellular MHT and intracellular MHT groups were directly injected with MNPs (Resovist $^{\oplus}$, FUJIFILM RI Pharma Co., Ltd., Tokyo, Japan) ( $0.2 \mathrm{~mL}$ of stock solution $(500 \mathrm{mM})$ diluted in phosphate-buffered saline) with an iron concentration of $400 \mathrm{mM}$. Because the molar mass of iron $(\mathrm{Fe})$ is $55.8 \mathrm{~g} / \mathrm{mol}$, the iron concentration of $400 \mathrm{mM}$ corresponds to $22.3 \mathrm{mg} \mathrm{Fe} / \mathrm{mL}$, and the total dose of MNPs administered was $4.46 \mathrm{mg}$ Fe. The mean and SD of the particle size of Resovist ${ }^{\oplus}$ are reported to be $15.2 \mathrm{~nm}$ and $3.21 \mathrm{~nm}$, respectively [16]. The zeta potential is -15 $\mathrm{mV}$ [17].

Figure 1(a) and Figure 1(b) illustrate the protocols for data acquisition in the extracellular MHT and intracellular MHT groups, respectively. In the extracellular MHT group (Figure 1(a)), MHT was started 15 min after the injection of MNPs and was performed by applying an AMF at a frequency of $600 \mathrm{kHz}$ and a peak amplitude of $3.1 \mathrm{kA} / \mathrm{m}$ [4] [5] for $20 \mathrm{~min}$. During MHT, the temperatures of the tumor and rectum were measured in 3 mice using two fluorescence-type optical fiber thermometers (AMOTH FL-2000, Anritsu Meter Co., Tokyo, Japan) and two optical fiber temperature probes. One probe was inserted into the center of the tumor and the other probe was inserted $1 \mathrm{~cm}$ inside the rectum. Both temperatures were recorded every second until the end of MHT. The temperature rise of the tumor was calculated from the temperature of the tumor minus that of the rectum. The MPI studies were performed using our MPI scanner [12] [13] 13 min before MHT (2 min after the injection of MNPs), 22 


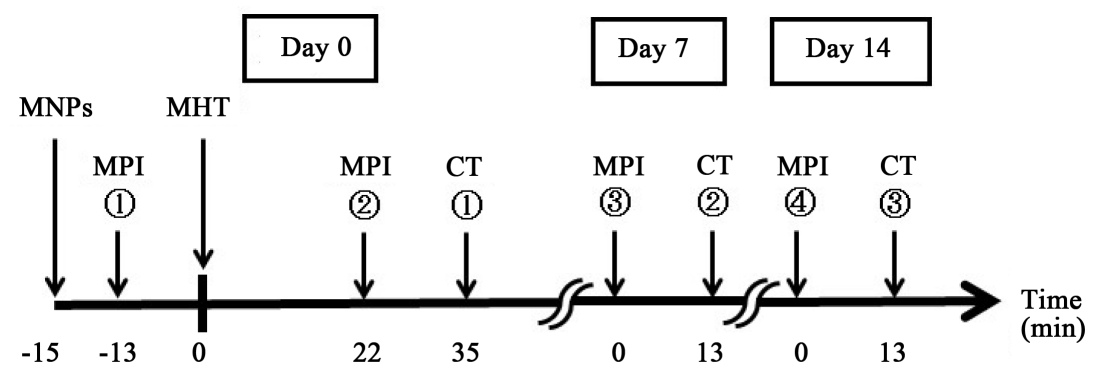

(a)

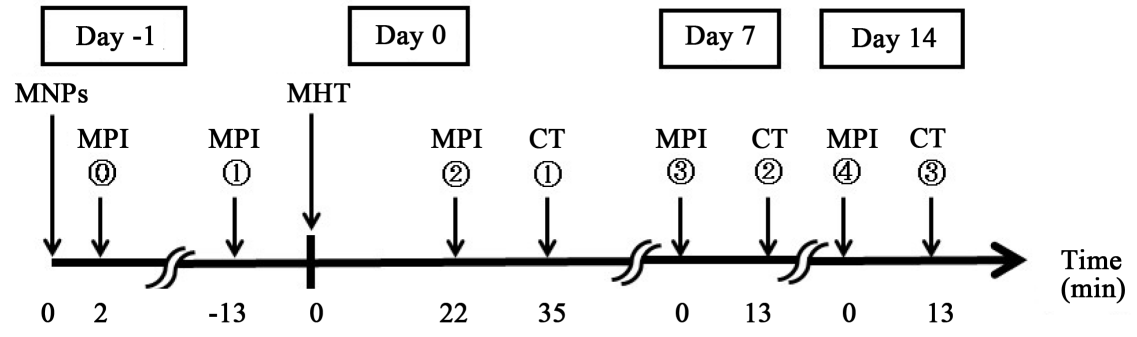

(b)

Figure 1. Protocol for data acquisition in the present study. (a) For extracellular magnetic hyperthermia treatment (MHT) and (b) for intracellular MHT. MNPs: magnetic nanoparticles, MPI: magnetic particle imaging, and CT: X-ray computed tomography. We used Resovist ${ }^{\oplus}$ as the source of MNPs and injected it directly into the tumor.

min after MHT (37 min after the injection of MNPs), and 7 and 14 days after MHT. After the second MPI study (35 min after MHT), X-ray CT images were obtained using a 4-row multi-slice CT scanner (Asteion, Toshiba Medical Systems Co., Tochigi, Japan) with a tube voltage of $120 \mathrm{kV}$, a tube current of 210 $\mathrm{mA}$, and a slice thickness of $0.5 \mathrm{~mm}$. The X-ray CT images were also acquired 7 and 14 days after MHT. The X-ray CT image after the second MPI study was substituted by that obtained after the first MPI study. The MPI images were coregistered to the X-ray CT images for anatomical identification using the method reported in [14] [15].

In the intracellular MHT group (Figure 1(b)), MHT was performed one day after the injection of MNPs in the same manner as for the extracellular MHT group. The temperatures of the tumor and rectum were also measured during MHT in 4 mice and the temperature rise of the tumor was calculated in the same manner as for the extracellular MHT group. The MPI studies were performed 2 min after the injection of MNPs, 13 min before MHT, 22 min after MHT, and 7 and 14 days after MHT. As in the extracellular MHT group, the X-ray CT images were acquired $35 \mathrm{~min}, 7$ days, and 14 days after MHT.

\subsection{Transmission Electron Microscopic Study}

Separately from the above studies, mice were purchased for transmission electron microscopic (TEM) studies and were implanted with colon-26 cells in the same manner as described above. When the tumor volume reached approximately $100 \mathrm{~mm}^{3}$, the mice were divided into extracellular MHT $(\mathrm{n}=3)$ and 
intracellular MHT groups $(\mathrm{n}=4)$. The mice were sacrificed and the tumors were removed immediately $(15 \mathrm{~min}$ ) and one day after the injection of MNPs in the extracellular MHT (n = 3) and intracellular MHT groups $(\mathrm{n}=4)$, respectively.

The resected tumor tissues were fixed in $7.5 \%$ formaldehyde neutral buffered solution (Sigma-Aldrich Japan Co., Ltd., Tokyo, Japan) and TEM observation was performed under an electron microscope (H-7650, Hitachi Ltd., Tokyo, Japan). The resolution of the electron microscope is $0.2 \mathrm{~nm}$ for lattice image.

\subsection{Data and Statistical Analyses}

The vertical diameter $\left(L_{x}\right)$, horizontal diameter $\left(L_{y}\right)$, and height $\left(L_{z}\right)$ of the tumor were measured with a caliper every day and the tumor volume ( $V$ ) was calculated from $V=(\pi / 6) \times L_{x} \times L_{y} \times L_{z}$ The relative tumor volume growth (RTVG) was also calculated from $\left(V-V_{0}\right) / V_{0}$. In the control group, $V_{0}$ was taken as the tumor volume immediately after it reached approximately $100 \mathrm{~mm}^{3}$. In the extracellular MHT and intracellular MHT groups, $V_{0}$ was taken as the tumor volume immediately before MHT.

After the MPI studies, we drew a region of interest (ROI) on the tumor in the MPI image (Figure 2) and calculated the average and maximum MPI values in the same manner as that in our previous papers [14] [15]. We also calculated the number of pixels within the ROI and then calculated the total MPI value from the product of the average MPI value and the number of pixels.

The temperature, temperature rise, RTVG, average MPI value, maximum MPI value, total MPI value, and the number of pixels within the ROI were expressed as the mean \pm standard error (SE). Differences in the RTVG value among groups were analyzed by one-way analysis of variance (ANOVA). Statistical significance was determined by Tukey-Kramer's multiple comparison test. Differences in the temperature and temperature rise of the tumor between the extracellular MHT and intracellular MHT groups were analyzed by Student's $t$-test. Differences in the average, maximum, and total MPI values and the number of pixels between the extracellular MHT and intracellular MHT groups were also analyzed by

(rel. unit)

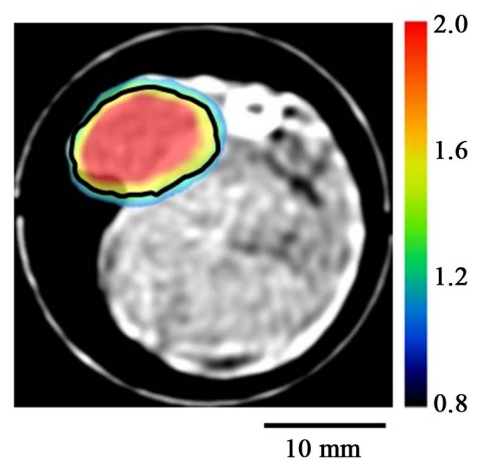

Figure 2. Example of an MPI image superimposed on an Xray $\mathrm{CT}$ image and the region of interest (ROI) drawn on the MPI image (black solid line). Scale bar $=10 \mathrm{~mm}$. 
Student's $t$-test. A $p$ value less than 0.05 was considered statistically significant.

\section{Results}

Figure 3 shows typical TEM images in the extracellular MHT (a) and intracellular MHT groups (b). In the extracellular MHT group, almost all the MNPs were aggregated in the extracellular space (arrows in Figure 3(a)), whereas they were contained within the intracellular space (arrows in Figure 3(b)) in the intracellular MHT group.

Figure 4(a) shows the time courses of the temperature of the tumor in the

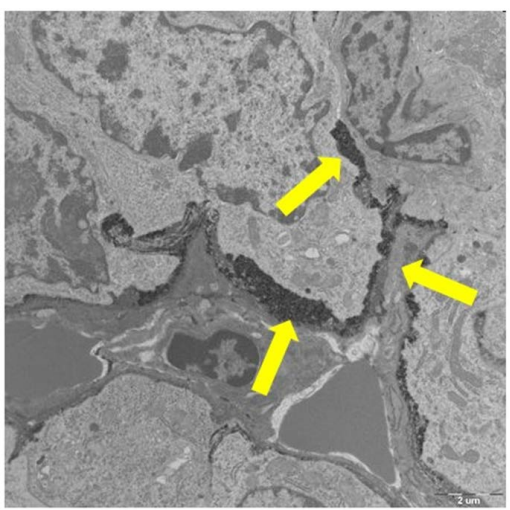

(a)

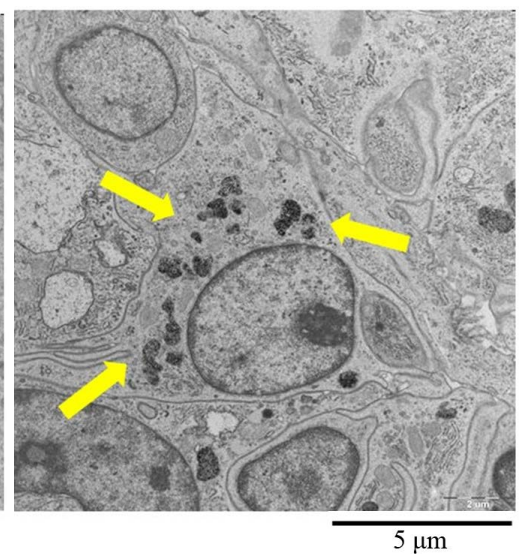

(b)

Figure 3. Transmission electron microscopic (TEM) images in the extracellular MHT (a) and intracellular MHT groups (b); arrows show MNPs. Scale bar $=5 \mu \mathrm{m}$.

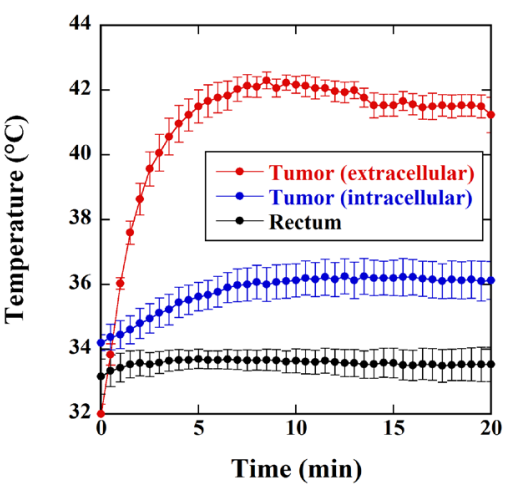

(a)

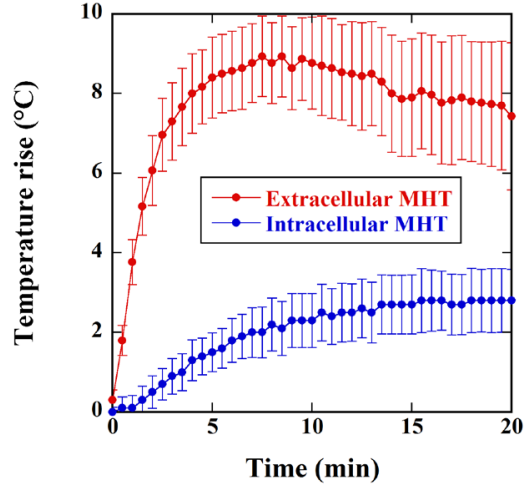

(b)

Figure 4. (a) Temperature of the tumor in the extracellular MHT (red circles, $n=3$ ) and intracellular MHT groups (blue circles, $\mathrm{n}=4$ ) and that of the rectum in both groups (black circles, $\mathrm{n}=7$ ). Data are presented as the mean \pm standard error (SE). The temperature of the tumor in the extracellular MHT group is significantly higher than that in the intracellular MHT group 1 min or more after the start of MHT; (b) Temperature rise (temperature of the tumor minus that of the rectum) in the extracellular MHT (red circles, $n=3$ ) and intracellular MHT groups (blue circles, $\mathrm{n}=4$ ). Data are presented as the mean \pm SE. The temperature rise of the tumor in the extracellular MHT group is significantly higher than that in the intracellular MHT group 30 s or more after the start of MHT. 
extracellular MHT (red circles, $\mathrm{n}=3$ ) and intracellular MHT groups (blue circles, $\mathrm{n}=4$ ) and that of the rectum in both groups (black circles, $\mathrm{n}=7$ ), whereas Figure 4(b) shows the time courses of the temperature rise of the tumor, i.e., the temperature of the tumor minus that of the rectum, in the extracellular MHT (red circles, $\mathrm{n}=3$ ) and intracellular MHT groups (blue circles, $\mathrm{n}=4$ ). The temperature of the tumor in the extracellular MHT group was significantly higher than that in the intracellular MHT group 1 min or more after the start of MHT (Figure 4(a)). Although the error range was large due to a small number of data, the temperature rise in the extracellular MHT group was significantly higher than that in the intracellular MHT group $30 \mathrm{~s}$ or more after the start of MHT (Figure 4(b)).

Figure 5 shows the average MPI value (a), maximum MPI value (b), total MPI value (c), and the number of pixels within the ROI (d) 2 min (MPI (a) in Figure 1(b)) and one day after the injection of MNPs (MPI (1) in Figure 1(b)). As shown in Figure 5, the average, maximum, and total MPI values significantly decreased by one day after the injection of MNPs, whereas the number of pixels within the ROI significantly increased.

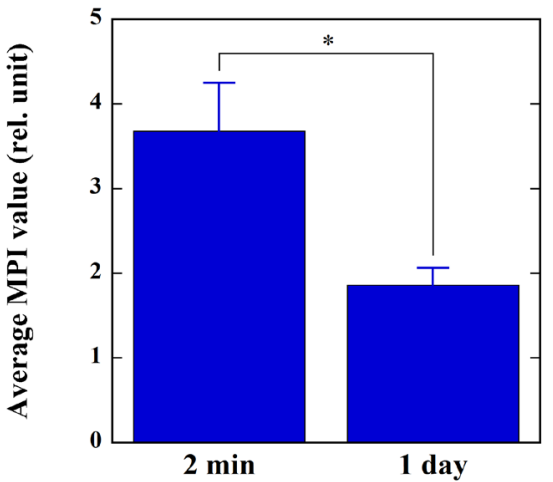

(a)

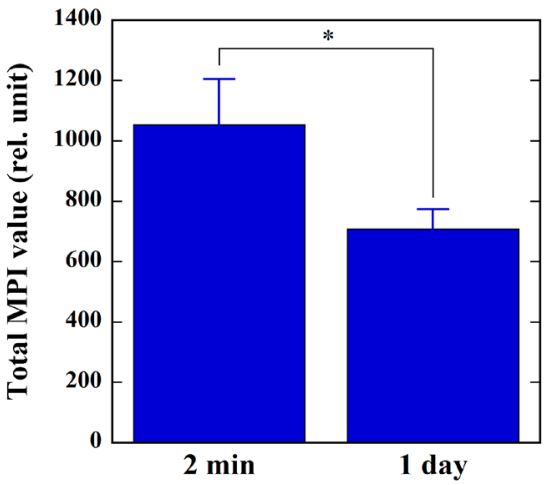

(c)

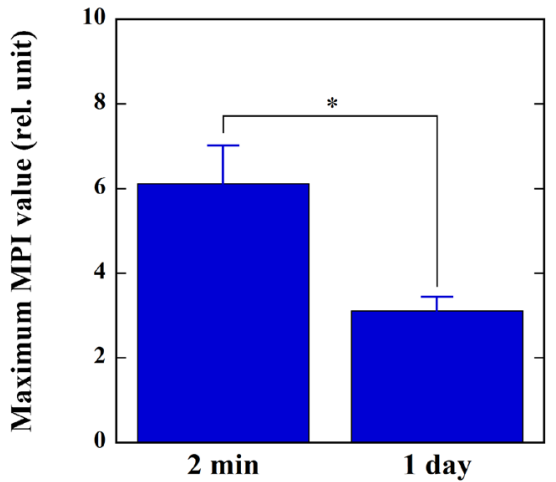

(b)

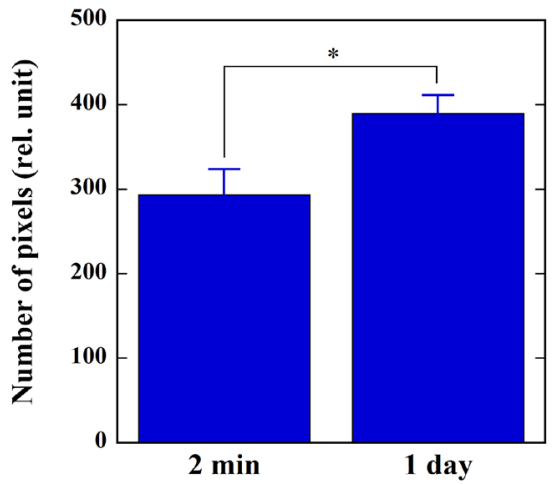

(d)

Figure 5. Average MPI value (a); maximum MPI value (b); total MPI value (c); and the number of pixels within the ROI (d) at 2 min (MPI (0) in Figure 1(b)) and one day after the injection of MNPs (MPI (1) in Figure 1(b)). The total MPI value is equal to the product of the average MPI value and the number of pixels. Bar and error bar represent the mean and SE, respectively. ${ }^{*}: p<0.05$. 
Figure 6 shows the temporal changes of the average MPI value (a), maximum MPI value (b), total MPI value (c), and the number of pixels within the ROI (d) in the extracellular MHT (red bars, $n=8$ ) and intracellular MHT groups (blue bars, $n=7$ ). It should be noted that the values immediately before MHT (MPI (1) in Figure 1) were normalized as unity. The normalized average MPI value in the intracellular MHT group was significantly higher than that in the extracellular MHT group immediately and 7 days after MHT (Figure 6(a)). Although there was a tendency for the normalized average MPI value in the intracellular MHT group to be higher than that in the extracellular MHT group 14 days after MHT, it did not reach statistical significance $(p=0.079)$. The normalized maximum MPI value in the intracellular MHT group was significantly higher than that in the extracellular MHT group 7 days after MHT (Figure 6(b)). Although the normalized maximum MPI value in the intracellular MHT group tended to be higher than that in the extracellular MHT group 14 days after MHT, it did not reach statistical significance $(p=0.080)$. The normalized total MPI value in

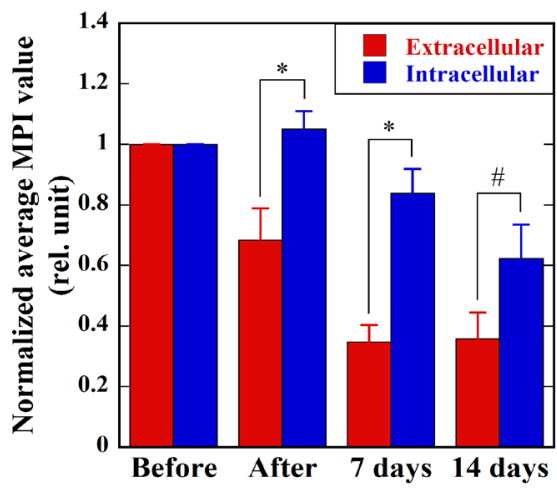

(a)

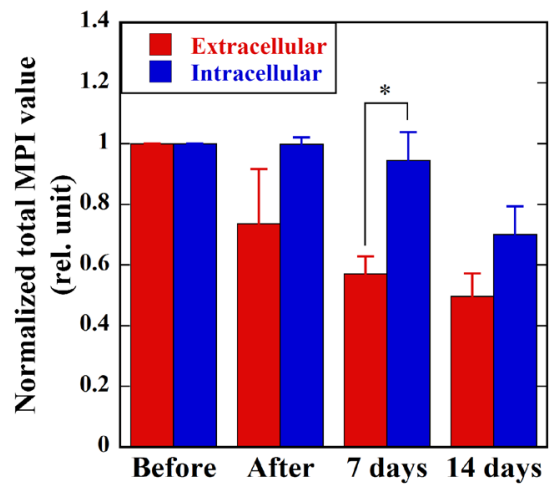

(c)

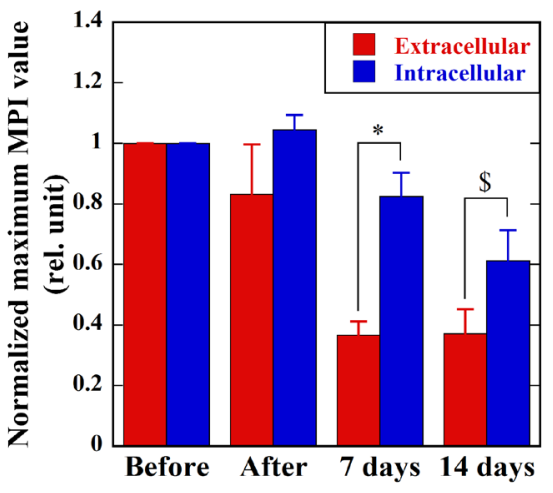

(b)

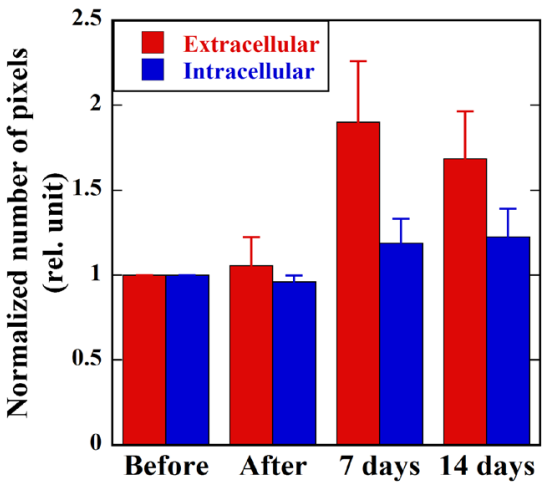

(d)

Figure 6. Normalized average MPI value (a); normalized maximum MPI value (b); normalized total MPI value (c); and normalized number of pixels within the ROI (d) immediately (13 min, MPI (1) in Figure 1) before, immediately (22 min, MPI (2) in Figure 1) after, 7 days (MPI (3) in Figure 1), and 14 days (MPI (4) in Figure 1) after MHT in the extracellular MHT (red bars, $\mathrm{n}=8$ ) and intracellular MHT groups (blue bars, $\mathrm{n}=7$ ). The values immediately before MHT were normalized as unity. Bar and error bar represent the mean and SE, respectively. ${ }^{\star}: p<0.05 . \#: p=0.079$. $\$: p=0.080$. 
the intracellular MHT group was significantly higher than that in the extracellular MHT group 7 days after MHT (Figure 6(c)). In contrast, the normalized number of pixels within the ROI in the intracellular MHT group tended to be lower than that in the extracellular MHT group (Figure 6(d)). This tendency, however, did not reach statistical significance.

Figure 7 shows the time courses of the RTVG value in the extracellular MHT (red circles, $\mathrm{n}=8$ ), intracellular MHT (blue circles, $\mathrm{n}=7$ ), and control groups (black circles, $\mathrm{n}=10$ ). The RTVG value in the extracellular MHT group was significantly lower than that in the control group 3 days or more after MHT. The RTVG value in the intracellular MHT group was significantly lower than that in the control group 2 days or more after MHT and it was significantly lower than that in the extracellular MHT group 3, 4, and 5 days after MHT.

\section{Discussion}

Our TEM studies demonstrated that almost all the MNPs were aggregated in the extracellular space immediately $(15 \mathrm{~min})$ after the intratumoral injection of MNPs, whereas they were contained within the intracellular space one day after the injection of MNPs (Figure 3). This is the reason why we referred to the MHT performed immediately (15 min) and one day after the injection of MNPs as "extracellular MHT" and "intracellular MHT", respectively. Giustini et al. [18] used TEM to evaluate the time-dependent cellular uptake of intratumorally injected dextran-coated MNPs with a mean hydrodynamic diameter of 100 to 130 $\mathrm{nm}$ and pointed out that intracellular uptake of the MNPs was 5.0\%, 48.8\%, and $91.8 \%$ uptake at 1, 2, and $4 \mathrm{~h}$ post injection of MNPs. The MNPs were contained

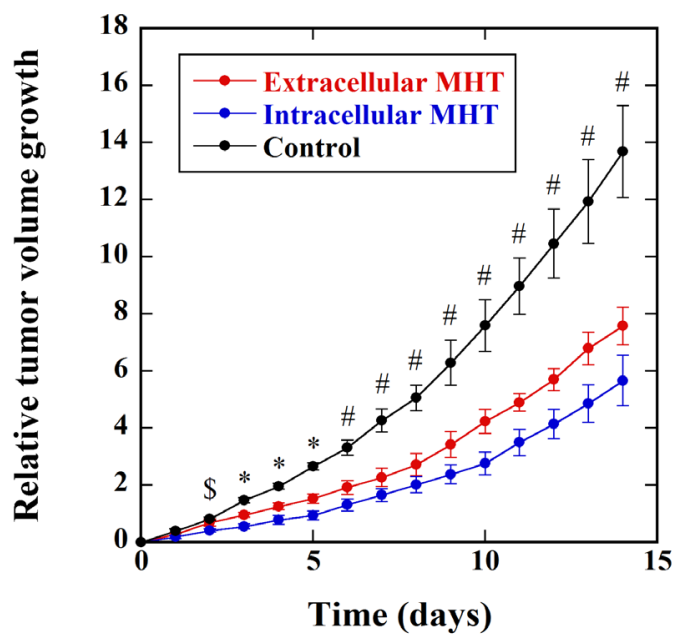

Figure 7. Relative tumor volume growth (RTVG) values in the extracellular MHT (red circles, $\mathrm{n}=8$ ), intracellular MHT (blue circles, $\mathrm{n}=7$ ), and control groups (black circles, $\mathrm{n}$ $=10) .{ }^{\star}: p<0.05$ between the control and extracellular MHT groups, between the control and intracellular MHT groups, and between the extracellular and intracellular MHT groups. \#: $p<0.05$ between the control and extracellular MHT groups and between the control and intracellular MHT groups. $\$: p<0.05$ between the control and intracellular MHT groups. 
within intracellular intracytoplasmic vesicles at one day after the injection of MNPs into the tumors [18]. Thus, our results (Figure 3) are consistent with the findings reported by Giustini et al. [18].

We compared the therapeutic effects of the extracellular MHT and intracellular MHT using the RTVG value (Figure 7). Although the temperature of the tumor in the intracellular MHT group was significantly lower than that in the extracellular MHT group (Figure 4), the RTVG value in the intracellular MHT group was significantly lower than that in the extracellular MHT group 3, 4, and 5 days after MHT (Figure 7), suggesting that intracellular MHT is more cytotoxic than extracellular MHT in spite of the significantly lower temperature of the tumor. It has been reported several times that hyperthermia-induced cell death can occur under a negligible temperature rise in vitro [6] [9]. To our knowledge, this study is the first report to show the superiority of intracellular MHT over extracellular MHT in vivo and that hyperthermia-induced cell death can also occur without a significant global increase in temperature in vivo.

To investigate the temporal change of intratumorally injected MNPs during one day, we calculated the average, maximum, and total MPI values and the number of pixels within the ROI (Figure 2) and compared these values at $2 \mathrm{~min}$ and one day after the injection of MNPs (Figure 5). As shown in Figure 5, the average, maximum, and total MPI values significantly decreased by almost half by one day after the injection of MNPs, whereas the number of pixels within the ROI significantly increased. As we previously reported, there was an excellent linear correlation between the average MPI value and the iron concentration of Resovist $^{\circledast}$ in phantom studies [14]. From this finding, the change in the average MPI value appears to correspond to that in the average amount of MNPs per voxel, i.e., the average concentration of MNPs, and the change in the total MPI value appears to correspond to that in the total amount of MNPs in the selected slice of the tumor, whereas the change in the number of pixels appears to correspond to that in the distributed area of MNPs. Thus, the results shown in Figure 5 appear to be mainly due to the fact that the MNPs injected into the tumor dispersed within the tumor during one day by diffusion and/or perfusion. This also appears to be the main reason why the temperature of the tumor in the intracellular MHT group was significantly lower than that in the extracellular MHT group (Figure 4).

Furthermore, we investigated the temporal change of the MNPs injected into the tumor by calculating the average, maximum, and total MPI values and the number of pixels within the ROI immediately before, immediately after, and 7 and 14 days after MHT and normalizing those immediately before MHT as unity (Figure 6). As shown in Figure 6(a), the normalized average MPI value in the intracellular MHT group was significantly higher than that in the extracellular MHT group immediately and 7 days after MHT. The normalized maximum and total MPI values in the intracellular MHT group were significantly higher than those in the extracellular MHT group 7 days after MHT (Figure 6(b) and Figure 
6(c)). In addition, the temporal changes of the above parameters in the intracellular MHT group were smaller than those in the extracellular MHT group (Figure 6). As suggested by our TEM studies (Figure 3), this appears to be mainly due to the fact that once MNPs were internalized by tumor cells, their dispersion within the tumor and/or to the outside of the tumor is more likely to be reduced than that of the MNPs located outside the cells. This feature of the intracellular MHT would be useful especially when considering repeated applications of MHT.

As previously described, our results (Figure 4 and Figure 7) demonstrated that hyperthermia-induced cell death can occur without a significant global increase in temperature in vivo. Several hypotheses have been proposed to explain the cytotoxicity in the intracellular MHT. One of the leading hypotheses is based on recent experiments showing that the temperature rise is localized in the very close vicinity of the MNP surface. Riedinger et al. [19] experimentally demonstrated using a molecular temperature probe that the local temperature induced by AMF increased by several tens of degree at distances below $0.5 \mathrm{~nm}$ from the MNP surface and decayed exponentially with increasing distance. Furthermore, significant local-to-global temperature differences could be found at distances shorter than $3 \mathrm{~nm}$ [19]. This local heating could damage the surfaces of lysosomes where MNPs are stored and/or catalyze a chemical reaction in lysosomes such as the Fenton reaction [20]. Our results suggest that the local temperature rise in cancer cells is more important rather than the global temperature rise for predicting the therapeutic effect of MHT and/or designing optimal treatment planning using MHT. In this respect, the present study offers important information regarding monitoring the temperature of the tumor during MHT.

We previously described that the average MPI value decreased by almost half during one day after the injection of MNPs (Figure 5). This may suggest that almost half of the MNPs dispersed within the tumor before being internalized by the cells. Thus, it would be necessary to develop a method for facilitating the rapid internalization of as many MNPs as possible into cancer cells in order to enhance the effectiveness of intracellular MHT. Thu et al. [21] reported a new straightforward magnetic cell-labeling approach that combines ferumoxytol, heparin, and protamine. Heparin and protamine form nanocomplexes through electrostatic interactions [21]. Combining heparin, protamine, and ferumoxytol results in the formation of a self-assembling nanocomplex that allows the rapid internalization of MNPs into cells [21]. Thus, this method appears to be a promising approach for enhancing the effectiveness of intracellular MHT. We are currently planning to use these approaches.

A limitation of this study is that the analysis of the temporal change of MNPs in the tumor was based on data obtained from a single slice of the MPI image. This use of a single slice of the MPI image limits the accurate evaluation of the concentration and spatial distribution of MNPs in the whole tumor. To overcome such a limitation, it will be necessary to acquire three-dimensional data and to evaluate the three-dimensional distribution and accumulation of MNPs 
from these data. In addition, we directly injected Resovist ${ }^{\oplus}$ into the tumor in this study, however, a method for active tumor-specific targeting by MNPs injected intravenously should be established for clinical application. We are also currently planning these studies.

Another limitation of this study is that the cytotoxicity of Resovist ${ }^{\otimes}$ against colon-26 cells has not been investigated using cytotoxicity assays such as the tetrazolium (MTT) assay [22]. Yeh et al. investigated the viability and cytotoxicity in macrophages treated with $200 \mu \mathrm{g} \mathrm{Fe} / \mathrm{mL}$ of Resovist ${ }^{\circledR}$ using the MTT assay and reported that they were not significantly affected [23]. However, macrophages loaded with Resovist ${ }^{\oplus}$ above $100 \mu \mathrm{g} \mathrm{Fe} / \mathrm{mL}$ showed a significant increase in cytokine secretion and mRNA expression [23]. Thus, it would be necessary to perform further in-vitro studies on the cytotoxicity of Resovist ${ }^{\circledR}$ against colon-26 cells in order to elucidate the reason for the present results (Figure 7). These studies are currently in progress.

\section{Conclusion}

Our results suggest that intracellular MHT is more cytotoxic than extracellular MHT inspite of the lower temperature rise of tumors, and that MPI is useful for evaluating the difference in temporal change of MNPs in the tumor between extracellular MHT and intracellular MHT.

\section{Acknowledgements}

This work was supported by JSPS KAKENHI Grant Numbers JP25282131 and JP15K12508.

\section{References}

[1] Rosensweig, R.E. (2002) Heating Magnetic Fluid with Alternating Magnetic Field. Journal of Magnetism and Magnetic Materials, 252, 370-374. https://doi.org/10.1016/S0304-8853(02)00706-0

[2] Petryk, A.A., Giustini, A.J., Gottesman, R.E., Trembly, B.S. and Hoopes, P.J. (2013) Comparison of Magnetic Nanoparticle and Microwave Hyperthermia Cancer Treatment Methodology and Treatment Effect in a Rodent Breast Cancer. International Journal of Hyperthermia, 29, 819-827. https://doi.org/10.3109/02656736.2013.845801

[3] Abe, M., Hiraoka, M., Takahashi, M., Egawa, S., Matsuda, C., Onoyama, Y., Morita, K., Kakehi, M. and Sugahara, T. (1986) Multi-Institutional Studies on Hyperthermia Using an 8-MHz Radiofrequency Capacitive Heating Device (Thermotron RF-8) in Combination with Radiation for Cancer Therapy. Cancer, 58, 1589-1595. https://doi.org/10.1002/1097-0142(19861015)58:8<1589::AID-CNCR2820580802>3. $\underline{0 . \mathrm{CO} ; 2-\mathrm{B}}$

[4] Kuboyabu, T., Yamawaki, M., Aoki, M., Ohki, A. and Murase, K. (2016) Quantitative Evaluation of Tumor Early Response to Magnetic Hyperthermia Combined with Vascular Disrupting Therapy Using Magnetic Particle Imaging. International Journal of Nanomedicine and Nanosurgery, 2, 1-7.

[5] Ohki, A., Kuboyabu, T., Aoki, M., Yamawaki, M. and Murase, K. (2016) Quantita- 
tive Evaluation of Tumor Response to Combination of Magnetic Hyperthermia Treatment and Radiation Therapy Using Magnetic Particle Imaging. International Journal of Nanomedicine and Nanosurgery, 2, 1-6.

[6] Villanueva, A., de la Presa, P., Alonso, J.M., Rueda, T., Martínez, A., Crespo, P., Morales, M.P., Gonzalez-Fernandez, M.A., Valdés, J. and Rivero, G. (2010) Hyperthermia HeLa Cell Treatment with Silica-Coated Manganese Oxide Nanoparticles. Journal of Physical Chemistry C, 114, 1976-1981. https://doi.org/10.1021/jp907046f

[7] Creixell, M., Bohorquez, A.C., Torres-Lugo, M. and Rinaldi, C. (2011) EGFR-Targeted Magnetic Nanoparticle Heaters Kill Cancer Cells without a Perceptible Temperature Rise. ASC Nano, 5, 7124-7139. https://doi.org/10.1021/nn201822b

[8] Grazu, V., Silber, A.M., Moros, M., Torres, T.E., Marquina, C., Ibarra, M.R. and Goya, G.F. (2012) Application of Magnetically Induced Hyperthermia in the Model Protozoan Crithidia Fasciculata as a Potential Therapy against Parasitic Infections. International Journal of Nanomedicine, 7, 5351-5360.

[9] de Sousa, M.E., Carrea, A., Zelis, P.M., Muraca, D., Mykhaylyk, O., Sosa, Y.E., Goya, R.G., Sánchez, F.H., Dewey, R.A. and van Raap, M.B.F. (2016) Stress-Induced Gene Expression Sensing Intracellular Heating Triggered by Magnetic Hyperthermia. Journal of Physical Chemistry C, 120, 7339-7348. https://doi.org/10.1021/acs.jpcc.5b12330

[10] Munoz-Menendez, C., Conde-Leboran, I., Serantes, D., Chantrell, R., Chubykalo-Fesenko, O. and Baldomir, D. (2016) Distinguishing between Heating Power and Hyperthermia Cell-Treatment Efficacy in Magnetic Fluid Hyperthermia. Soft Matter, 12, 8815-8818. https://doi.org/10.1039/C6SM01910B

[11] Gleich, B. and Weizenecker, J. (2005) Tomographic Imaging Using the Nonlinear Response of Magnetic Particles. Nature, 435, 1214-1217.

https://doi.org/10.1038/nature03808

[12] Murase, K., Hiratsuka, S., Song, R. and Takeuchi, Y. (2014) Development of a System for Magnetic Particle Imaging Using Neodymium Magnets and Gradiometer. Japanese Journal of Applied Physics, 53, Article ID: 067001. https://doi.org/10.7567/JJAP.53.067001

[13] Murase, K., Song, R. and Hiratsuka, S. (2014) Magnetic Particle Imaging of Blood Coagulation. Applied Physics Letters, 104, Article ID: 252409. https://doi.org/10.1063/1.4885146

[14] Murase, K., Aoki, M., Banura, N., Nishimoto, K., Mimura, A., Kuboyabu, T. and Yabata, I. (2015) Usefulness of Magnetic Particle Imaging for Predicting the Therapeutic Effect of Magnetic Hyperthermia. Open Journal of Medical Imaging, 5, 85-99. https://doi.org/10.4236/ojmi.2015.52013

[15] Kuboyabu, T., Yabata, I., Aoki, M., Banura, N., Nishimoto, K., Mimura, A. and Murase, K. (2016) Magnetic Particle Imaging for Magnetic Hyperthermia Treatment: Visualization and Quantification of the Intratumoral Distribution and Temporal Change of Magnetic Nanoparticles in Vivo. Open Journal of Medical Imaging, 6, 1-15. https://doi.org/10.4236/ojmi.2016.61001

[16] Biederer, S., Knopp, T., Sattel, T.F., Ludtke-Buzug, K., Gleich, B., Weizenecker, J., Borgert, J. and Buzug, T.M. (2009) Magnetization Response Spectroscopy of Superparamagnetic Nanoparticles for Magnetic Particle Imaging. Journal of Physics D: Applied Physics, 42, Article ID: 205007. https://doi.org/10.1088/0022-3727/42/20/205007

[17] Oishi, K., Miyamoto, Y., Saito, H., Murase, K., Ono, K., Sawada, M., Watanabe, M., Noguchi, Y., Fujiwara, T., Hayashi, S. and Noguchi, H. (2013) In Vivo Imaging of 
Transplanted Islets Labeled with a Novel Cationic Nanoparticle. Plos One, 8, Article ID: e57046. https://doi.org/10.1371/journal.pone.0057046

[18] Giustini, A.J., Ivkov, R. and Hoopes, P.J. (2011) Magnetic Nanoparticle Biodistribution Following Intratumoral Administration. Nanotechnology, 22, Article ID: 345101. https://doi.org/10.1088/0957-4484/22/34/345101

[19] Riedinger, A., Guardia, P., Curcio, A., Garcia, M.A., Cingolani, R., Manna, L. and Pellegrino, T. (2013) Subnanometer Local Temperature Probing and Remotely Controlled Drug Release Based on Azo-Functionalized Iron Oxide Nanoparticles. Nano Letters, 13, 2399-2406. https://doi.org/10.1021/nl400188q

[20] Hallali, N., Cierc, P., Fourmy, D., Gigoux, V. and Carrey, J. (2016) Influence on Cell Death of High Frequency Motion of Magnetic Nanoparticles during Magnetic Hyperthermia Experiments. Applied Physics Letters, 109, Article ID: 032402. https://doi.org/10.1063/1.4958989

[21] Thu, M.S., Bryant, L.H., Coppola, T., Jordan, E.K., Budde, M.D., Lewis, B.K., Chaudhry, A., Ren, J., Varma, N.R., Arbab, A.S. and Frank, J.A. (2012) Self-Assembling Nanocomplexes by Combining Ferumoxytol, Heparin and Protamine for Cell Tracking by Magnetic Resonance Imaging. Nature Medicine, 18, 463-467. https://doi.org/10.1038/nm.2666

[22] Mosmann, T. (1983) Rapid Colorimetric Assay for Cellular Growth and Survival: Application to Proliferation and Cytotoxicity Assays. Journal of Immunological Methods, 65, 55-63. https://doi.org/10.1016/0022-1759(83)90303-4

[23] Yeh, C.H., Hsiao, J.K., Wang, J.L. and Sheu, F. (2010) Immunological Impact of Magnetic Nanoparticles (Ferucarbotran) on Murine Peritoneal Macrophages. Journal of Nanoparticle Research, 12, 151-160. https://doi.org/10.1007/s11051-009-9589-y 